\title{
Coberturas curtas em espaços de Hamming
}

\author{
Anderson N. Martinhão, Emerson L. Monte Carmelo \\ Departamento de Matemática, UEM, \\ 87020-900, Maringá, PR \\ E-mail: anderson.martinhao@gmail.com, elmcarmelo@uem.br
}

\begin{abstract}
Resumo: Seja $\mathbb{F}_{q}^{3}$ o espaço vetorial tridimensional sobre o corpo finito $\mathbb{F}_{q}$. No espaço métrico induzido pela distância de Hamming, a bola estendida é a união de todas as bolas de raio 1 com centros nos múltiplos escalares de u. O seguinte problema de cobertura é induzido: dizemos que um subconjunto $\mathcal{H}$ de $\mathbb{F}_{q}^{3}$ é uma cobertura curta se a união de todas as bolas estendidas com centros nos elementos de $\mathcal{H}$ cobrem todo o espaço. Neste trabalho, algumas condições necessárias e algumas condições suficientes para uma cobertura curta são discutidas. Também determinamos cardinalidade minima de uma cobertura curta para algumas instâncias de $q$.
\end{abstract}

Palavras-chave: Códigos, coberturas, distância de Hamming, corpos finitos, ações de grupos.

\section{Introdução}

\section{$1.1 \quad$ O problema clássico}

Algumas aplicações da teoria de códigos de cobertura são: compressão com distorção, compressão de dados, decodificação de erros, transmissão em redes interconectadas, codificação de voz, telecomunicação via celular e outros, conforme [3]. Conceitos de outras áreas da matemática, como álgebra, combinatória e teoria dos números têm sido aplicados a essa teoria. Para uma visão geral e algumas aplicações de códigos de coberturas veja o livro [3].

O problema de coberturas tem suas origens em 1948, quando Taussky e Todd [11] o introduziram no contexto da teoria de grupos. Com o passar dos anos o problema de coberturas ganhou um contexto mais combinatório e foi estudado dentro da teoria dos códigos, e tem se mostrado um grande desafio. Neste novo contexto, surgiram mais problemas, como por exemplo o problema de encontrar bons sistemas de loteria esportiva (football pool systems) enunciado a seguir. Dadas $n$ partidas de futebol a serem realizadas, deve-se determinar o menor número de apostas que devem ser feitas para garantir que exista pelo menos uma aposta que consiga acertar o resultado de pelo menos $n-1$ dessas partidas. Este problema da loteria esportiva vem sendo estudado desde os anos 50 por matemáticos, cientistas da computação e engenheiros. Alguns pesquisadores que estudaram esse problema são Kamps, Van Lint, Hämäläinen, Rankinen, Östergard. Além do Brasil, este sistema de loteria esportiva é comum em países escandinavos, como Suécia e Finlândia.

Seja $Q$ um conjunto finito com $q$ elementos para qualquer inteiro $q$. Para simplificar a notação utilizaremos $Q=\mathbb{Z}_{q}$ o anel dos inteiro módulo $q$. Uma estrutura de espaço métrico é induzida em $\mathbb{Z}_{q}^{n}$ quando considerada a distância de Hamming que é definida da seguinte maneira: dados $u$ e $v$ em $\mathbb{Z}_{q}^{n}, d(u, v)$ denota o número de coordenadas em que $u$ e $v$ diferem. Por exemplo, $d(000,102)=2$, pois estes vetores diferem na primeira e na terceira coordenadas. Um subconjunto $\mathcal{C}$ de $\mathbb{Z}_{q}^{n}$ é um código de cobertura, ou simplesmente uma $R$-cobertura quando a união das bolas com raio $R$, com centros nos vetores de $\mathcal{C}$ é todo o espaço. Uma pergunta interessante da teoria é: Qual é a cardinalidade mínima $K_{q}(n, R)$ de uma $R$-cobertura em $\mathbb{Z}_{q}^{n}$ ?

Com o passar dos anos, os cálculos revelaram-se extremamente difíceis. Usando várias ferramentas e introduzindo novos conceitos, poucas classes exatas desse problema extremal foram 
determinadas até hoje. Até meados da década de 80, esses tipos de problemas foram estudados considerando-se apenas o raio 1. Em $[1,2]$ Carnielli estendeu o estudo desses números para raios arbitrários $R$. Em alguns desses casos foram obtidas aproximações para estas classes de valores de $K_{q}(n, R)$.

É comum também buscar valores computacionalmente. Códigos de cobertura podem ser reformulados em termos de conjuntos dominantes em grafos, que é um problema NP-completo, de acordo com [4]. Uma tabela atualizada desses valores é mantida por Kéri em [5]. Atualmente, devido a grande dificuldade computacional, diversos tipos de coberturas têm sido investigadas na tentativa de fornecer informações.

\subsection{Uma variante: Coberturas curtas}

Este trabalho concentra-se no estudo de uma nova variante, recentemente introduzida por Monte Carmelo, Nakaoka e Gerônimo em [9]. Para que esse novo problema esteja bem definido é essencial usarmos o corpo $\mathbb{F}_{q}$, pois utilizaremos a multiplicação de um vetor por escalar, assim o número $q$ é uma potência de primo. Naturalmente quando $q$ for um número primo, usaremos o corpo $\mathbb{F}_{q}=\mathbb{Z}_{q}$ (aritmética modular).

Seja $\mathbb{F}_{q}^{n}$ o espaço vetorial sobre o corpo finito $\mathbb{F}_{q}$ e considere a seguinte mudança do ponto de vista "geométrico": cada centro $u$ é trocado pela linha $\left\{\lambda u: \lambda \in \mathbb{F}_{q}\right\}$. Mais especificamente, dado um vetor $u \in \mathbb{F}_{q}^{n}$, a bola estendida de centro $u$ e raio $R$ é definida como sendo a união de todas as bolas de raio $R$ e com centros nos múltiplos escalares de $u$, isto é,

$$
E(u, R)=\bigcup_{\lambda \in \mathbb{F}_{q}} B(\lambda u, R)
$$

onde $B(x, R)$ denota a bola no espaço de Hamming $\mathbb{F}_{q}^{n}$ de centro $x$ e raio $R$. Um subconjunto $\mathcal{H}$ de $\mathbb{F}_{q}^{n}$ é uma $R$-cobertura curta, se a união das bolas estendidas centradas nos vetores de $\mathcal{H}$ cobre todo o espaço $\mathbb{F}_{q}^{n}$. Analogamente ao problema clássico pode-se definir o seguinte problema extremal: Determinar a cardinalidade mínima de uma $R$-cobertura curta de $\mathbb{F}_{q}^{n}$. Esse número é denotado por $c_{q}(n, R)$.

Essa nova função está profundamente relacionada com a função clássica. Ainda no trabalho [9] foi provada a seguinte relação entre esses dois tipos de coberturas, a saber, para todos $n>R>0$,

$$
c_{q}(n, R)+1 \leq K_{q}(n, R) \leq(q-1) c_{q}(n, R)+1 .
$$

Vários resultados da função clássica foram transladados naturalmente para esse novo problema, e em [8], Mendes e outros provaram um resultado de coberturas (clássicas) via coberturas curtas. Foi provado $c_{5}(10,7)=2$ e, a partir desse resultado, pôde-se concluir que $K_{5}(10,7)=9$.

Considere partir de agora o caso particular em que $n=3$ e $R=1$. Por isso, denote simplesmente por $c(q)$ o número $c_{q}(3,1)$ e a bola estendida $E(u, 1)$ por $E(u)$ para cada $u \in \mathbb{F}_{q}^{3}$. Segundo [7] e suas referências, os melhores limites conhecidos são

$$
\left\lceil\frac{q+1}{2}\right\rceil \leq c(q) \leq\left\{\begin{array}{l}
(q+3) / 2, \text { se } q \equiv 3 \bmod 4 \\
(q+5) / 2, \text { se } q \equiv 1 \bmod 4 \\
3(q+4) / 4, \text { se } q \text { é par. }
\end{array}\right.
$$

Resultados de [7] foram obtidos a partir de emparelhamentos em grafos completos com pesos. Devido a dificuldade deste problema, são conhecidos apenas alguns valores para $c(q)$.

\begin{tabular}{|c|c|c|c|c|c|c|c|}
\hline$q$ & 2 & 3 & 4 & 5 & 7 & 8 & 9 \\
\hline$c(q)$ & 1 & 3 & 3 & 4 & $4-5$ & $5-9$ & $5-7$ \\
\hline
\end{tabular}

Como pode-se ver, alguns valores pequenos de $q$ ainda estão em aberto.

O principal objetivo deste trabalho é a busca por condições que ajudem a afirmar se um subconjunto $\mathcal{H}$ de $\mathbb{F}_{q}^{3}$ é uma cobertura curta ou não. Como consequência seguirá os valores 
exatos para os números $c(q)$ para algumas instâncias de $q$. Na Seção 2 encontra-se alguns resultados referentes a cardinalidade das bolas estendidas, que são úteis para o decorrer do trabalho. Na Seção 3 são provados os limites inferiores $c(7) \geq 5, c(8) \geq 6$ e $c(9) \geq 6$. Na Seção 4 coberturas curtas minimais para $\mathbb{F}_{q}^{3}$ são exibidas para alguns casos de $q$.

A maioria das provas serão omitidas. Para o leitor mais interessado, uma versão completa deste trabalho pode ser encontrado em [6].

\section{Preliminares}

No manuscrito [6], a fim de obter limitantes inferiores para a função $c(q)$, os autores investigam como as bolas estendidas se intersectam. Às vezes, uma bola estendida cobre boa parte do espaço, por exemplo em $\mathbb{F}_{5}^{3}$ temos que $|B(1,1,1)|=13,|E(1,1,1)|=65$, enquanto que $\mathbb{F}_{5}^{3}=125$. Além disso, um novo obstáculo surge nesse ponto: no caso clássico temos que as cardinalidade das bolas independem do centro, enquanto que $|E(u)|$ não são independentes do centro $u$, por exemplo, em $\mathbb{F}_{5}^{3},|E((0,0,0))|=|B((0,0,0))|=13$, enquanto que $|E((1,1,1))|=65$, veja [9].

A busca de limites inferiores é um problema bastante desafiador. Uma tática já usada para o caso clássico é estudar o comportamento das cardinalidades das bolas estendidas restritas a algum subconjunto particular do espaço $\mathbb{F}_{q}^{n}$. De modo natural essa estratégia pode ser usada também no caso de coberturas curtas. Considere o seguinte subconjunto de $\mathbb{F}_{q}^{3}$,

$$
\mathcal{D}_{q}=\left\{\left(u_{1}, u_{2}, u_{3}\right) \in \mathbb{F}_{q}^{3}: u_{1}, u_{2}, u_{3} \text { são dois a dois distintos e não nulos }\right\}
$$

Denote a bola estendida $E(u)$ restrita a $\mathcal{D}_{q}$ por $\widetilde{E}(u)$. O número $|\widetilde{E}(u)|$ está determinado.

Teorema 1. [6, Teorema 5] Seja $u=\left(u_{1}, u_{2}, u_{3}\right)$ um vetor em $\mathbb{F}_{q}^{3}$, denote por $\omega(u)$ o peso do vetor $u$, isto é, $\omega(u)$ é a quantidade de coordenadas não nulas de u e por $\delta(u)=\left|\left\{u_{1}, u_{2}, u_{3}\right\}\right|$.

1. Se $\omega(u)=1$, então $|\widetilde{E}(u)|=0$.

2. Se $\omega(u)=2$, então $|\widetilde{E}(u)|=0$ ou $|\widetilde{E}(u)|=(q-1)(q-3)$, se $\delta(u)=1$ ou $\delta(u)=2$ respectivamente.

3. Se $\omega(u)=3$, então $|\widetilde{E}(u)|=0$ ou $|\widetilde{E}(u)|=(q-1)(2 q-6)$ ou $|\widetilde{E}(u)|=(q-1)(3 q-11)$, se $\delta(u)=1$ ou $\delta(u)=2$ ou $\delta(u)=3$ respectivamente.

É um pouco surpreendente que no cálculo de $|\widetilde{E}(u) \cap \widetilde{E}(v)|$, sob a condição $u, v \in \mathcal{D}_{q}$, o limite inferior dependa consideravelmente da forma aritmética de $q$, como vemos no próximo resultado.

Teorema 2. [6, Teorema 1] Dados q uma potência de primo e $u, v \in \mathcal{D}_{q}$, tem-se

$$
|\widetilde{E}(u) \cap \widetilde{E}(v)| \geq \begin{cases}2(q-1) & \text { se } q-1 \not \equiv 0 \\ 0 & \text { se } q-1 \equiv 0 \bmod 3\end{cases}
$$

Os limites são atingidos, isto é, se $q-1 \not \equiv 0 \bmod 3$, existem $u, v \in \mathcal{D}_{q}$ tais que $|\widetilde{E}(u) \cap \widetilde{E}(v)|=$ $2(q-1)$, e se $q-1 \equiv 0 \bmod 3$, existem $u, v \in \mathcal{D}_{q}$ tais que $|\widetilde{E}(u) \cap \widetilde{E}(v)|=0$.

\section{Condições necessárias}

Algumas condições necessárias para uma cobertura curta com poucos vetores são discutidas agora. Denote as projeções canônicas de $\mathbb{F}_{q}^{3}$ em $\mathbb{F}_{q}$, por $\pi_{i}\left(u_{1}, u_{2}, u_{3}\right)=u_{i}$, para cada $i \in\{1,2,3\}$. $\mathrm{O}$ símbolo $*$ representa um elemento arbitrário em $\mathbb{F}_{q}$.

Teorema 3. Sejam $q \geq 7$ uma potência de primo e $m=\lceil(q+1) / 2\rceil$. Suponha que $\mathcal{H}=$ $\left\{h_{1}, \ldots, h_{m}\right\}$ é uma cobertura curta de $\mathbb{F}_{q}^{3}$. As seguintes condições seguem: 
1. Existe um vetor em $\mathcal{H}$ com peso 3.

2. Para cada coordenada $j, 1 \leq j \leq 3$, existe um vetor $h_{k} \in \mathcal{H}$ tal que $\pi_{j}\left(h_{k}\right)=0$.

3. Podemos assumir sem perda de generalidade que $\mathcal{H}$ assume um das formas:

$$
\begin{aligned}
& \mathcal{H}_{1}=\left\{(1,1,1),(0, *, *),(*, 0, *),(*, *, 0), h_{5}, \ldots, h_{m}\right\} \\
& \mathcal{H}_{2}=\left\{(1,1,1),(0, *, *),(*, 0,0), h_{4}, \ldots, h_{m}\right\} .
\end{aligned}
$$

Ideia da demonstração. A demonstração da primeira parte deste teorema consiste em contar quantos elementos um vetor de peso no máximo 2 pode cobrir. Isso é feito aplicando o Teorema 1. Para provar a segunda parte, considera-se o plano $\Pi_{1}=\left\{\left(0, u_{2}, u_{3}\right): u_{2}, u_{3} \in \mathbb{F}_{q}\right\}$ e o seu subconjunto $\mathcal{X}_{1}=\left\{\left(0, u_{2}, u_{3}\right) \in \Pi_{1}: u_{2} \neq u_{3}\right.$ e $\left.u_{2}, u_{3} \neq 0\right\}$. A terceira parte é consequência imediata das duas primeiras partes. Ver detalhes em [6, Teorema 18].

\subsection{Aplicações numéricas}

O propósito do restante dessa seção é apresentar condições para se obter limites inferiores para $c(q)$ e também fornecer valores exatos pelo menos para $q \in\{7,8,9\}$. A condição $c(q)>m$ corresponde à afirmação: nenhum dos $\left(\begin{array}{c}q^{3} \\ m\end{array}\right) m$-subconjuntos $\mathcal{H}$ de $\mathbb{F}_{q}^{3}$ satisfazem

$$
\bigcup_{h \in \mathcal{H}} E(h)=\mathbb{F}_{q}^{3}
$$

Visto que o espaço é muito grande e as bolas estendidas são altamente intersectantes, não é fácil checar (3). A abordagem utilizada analisa essencialmente o comportamento das bolas estendidas restritas a $\mathcal{D}_{q}$. Mais precisamente, a ideia está descrita brevemente como segue.

Dado $q$ uma potência de primo, suponha por absurdo que existe uma cobertura curta $\mathcal{H}=$ $\left\{h_{1}, \ldots, h_{m}\right\}$ de $\mathbb{F}_{q}^{3}$ com $m=\lceil(q+1) / 2\rceil$ vetores. O Teorema 3 afirma que existem apenas duas possibilidades para $\mathcal{H}$. Visto que $\mathcal{H}$ é uma cobertura curta do subconjunto $\mathcal{D}_{q}$, a condição $\mathcal{D}_{q} \subset \cup_{i=1}^{m} \widetilde{E}\left(h_{i}\right)$ segue. Por outro lado, ao mostrar que

$$
\left|\bigcup_{i=1}^{m} \widetilde{E}\left(h_{i}\right)\right|<(q-1)(q-2)(q-3),
$$

tem-se uma contradição: $\mathcal{D}_{q}$ não está contido em $\cup_{i=1}^{m} \widetilde{E}\left(h_{i}\right)$, visto que $\left|\mathcal{D}_{q}\right|=(q-1)(q-2)(q-3)$.

Omitiremos algumas demonstrações dessa seção. Faremos com detalhes a demonstração do caso $q=7$.

Proposição 4. Obtemos $c(7) \geq 5$.

Demonstração. Suponha por absurdo que $\mathcal{H}=\left\{h_{1}, \ldots, h_{4}\right\}$ é uma cobertura curta de $\mathbb{F}_{7}^{3}$. O Teorema 3 implica que existem apenas duas possíveis formas para $\mathcal{H}$, a saber:

$$
\begin{aligned}
& \mathcal{H}_{1}=\{(1,1,1),(0, *, *),(*, 0, *),(*, *, 0)\}, \\
& \mathcal{H}_{2}=\{(1,1,1),(0, *, *),(*, 0,0),(*, *, *)\} .
\end{aligned}
$$

Se $\mathcal{H}=\mathcal{H}_{1}$, segue do Teorema $1,\left|\widetilde{E}\left(h_{1}\right)\right|=0$ e $\left|\widetilde{E}\left(h_{i}\right)\right| \leq 24$ para todo $i \in\{2,3,4\}$. Então $\mathcal{H}_{1}$ cobre no máximo 72 vetores de $\mathcal{D}_{7}$.

Se $\mathcal{H}=\mathcal{H}_{2}$, o Teorema 1 implica que $\left|\widetilde{E}\left(h_{1}\right)\right|=0,\left|\widetilde{E}\left(h_{2}\right)\right| \leq 24,\left|\widetilde{E}\left(h_{3}\right)\right|=0$ e $\left|\widetilde{E}\left(h_{4}\right)\right| \leq 60$. Então $\mathcal{H}_{2}$ cobre no máximo 84 vetores de $\mathcal{D}_{7}$.

Portanto, com $\left|\mathcal{D}_{7}\right|=120$, a desigualdade (4) segue, donde $\mathcal{H}$ não pode ser uma cobertura curta de todo o espaço $\mathbb{F}_{7}^{3}$. Assim, $c(7) \geq 5$. 
$\mathrm{O}$ argumento para $c(8)>5$ é o mais intrincado. O espaço de todas as possíveis coberturas curtas de $\mathbb{F}_{8}^{3}$ com 5 elementos, corresponde a família de todos os 5 -subconjuntos de $\mathbb{F}_{8}^{3}$, ou seja, são $\left(\begin{array}{c}8^{3} \\ 5\end{array}\right) \simeq 2.8 \times 10^{11}$ candidatos. O Teorema 1 não é suficiente para lidar com todos. Nesse caso aplica-se o Teorema 2 .

Proposição 5. O limite $c(8) \geq 6$ é válido.

Demonstração. A demonstração pode ser encontrada detalhada em [6, Proposição 21].

Proposição 6. O limite inferior $c(9) \geq 6$ segue.

Demonstração. Ver [6, Proposição 20].

\section{Condições suficientes}

\subsection{Ações de grupos aplicadas a coberturas curtas}

Um método de encontrar coberturas curtas é baseado em encontrar conjuntos que são invariantes sob certas ações de grupos.

Dado uma potência de primo $q, L_{q}$ denota o grupo de todos os operadores lineares nãosingulares $\mathbb{F}_{q}$, isto é,

$$
L_{q}=\left\{\sigma: \mathbb{F}_{q} \rightarrow \mathbb{F}_{q}: \sigma(x)=\lambda x, \text { para algum } \lambda \in \mathbb{F}_{q}^{*}\right\} .
$$

Seja $L_{q}^{3}$ o produto direto $L_{q} \oplus L_{q} \oplus L_{q}$. Como usual, $S_{3}$ denota o grupo simétrico de grau 3 . Considere o seguinte subgrupo de $L_{q}^{3}$,

$$
K=\left\{(\sigma, \sigma, \sigma): \sigma \in L_{q}\right\}=\left\{\left(u_{1}, u_{2}, u_{3}\right) \mapsto\left(\lambda u_{1}, \lambda u_{2}, \lambda u_{3}\right): \lambda \in \mathbb{F}_{q}^{*}\right\}
$$

A ação do produto direto $G=S_{3} \times K$ em $\mathbb{F}_{q}^{3}$ tem papel fundamental nos resultados, e para $(\varphi,(\sigma, \sigma, \sigma)) \in G$, e $u=\left(u_{1}, u_{2}, u_{3}\right) \in \mathbb{F}_{q}^{3}$, é dada por,

$$
u^{(\varphi,(\sigma, \sigma, \sigma))}=\left(\lambda u_{\varphi^{-1}(1)}, \lambda u_{\varphi^{-1}(2)}, \lambda u_{\varphi^{-1}(3)}\right) .
$$

Ou seja, o vetor $u$ é multiplicado por um escalar não nulo e tem as suas entradas permutadas. O conjunto

$$
\mathcal{A}_{q}=\left\{\left(u_{1}, u_{2}, u_{3}\right) \in \mathbb{F}_{q}^{3}: u_{1}, u_{2}, u_{3} \text { são dois a dois distintos }\right\}
$$

é invariante pela ação do produto direto $S_{3} \times K$, e tem duas órbitas, a saber, $\left\{u \in \mathcal{A}_{q}: d(u, 0)=\right.$ $3\}$ e $\left\{u \in \mathcal{A}_{q}: d(u, 0)=2\right\}$.

Um método de encontrar coberturas curtas é descrito em [10, Teorema 1]. Uma adaptação deste método é descrito abaixo.

Teorema 7. Seja $N$ um subgrupo de $S_{3}$ e escolha um subconjunto $\mathcal{L}$ de $\mathbb{F}_{q}^{3}$ que é invariante pela ação de $N$, isto é, $\mathcal{L}^{N}=\mathcal{L}$. Seja $\mathcal{O}$ a família de todas as órbitas da ação de $N \times K$ sobre $\mathcal{A}_{q}$. Suponha que cada órbita da ação de $S_{3} \times K$ em $\mathcal{A}_{q}$ contém um elemento u que pode ser escrito como $u=\lambda h+\mu e_{j}$ para algum $h \in \mathcal{L}, \lambda, \mu \in \mathbb{F}_{q}$ e $j \in\{1,2,3\}$. Então, o conjunto $\mathcal{L} \cup\{(1,1,1)\}$ é uma cobertura curta de $\mathbb{F}_{q}^{3}$.

Demonstração. Ver [6, Teorema 22]

O próximo exemplo mostra uma aplicação do método descrito acima para a construção de uma cobertura curta ótima para o caso $q=5$. 
Exemplo 8. O teorema acima é otimal para alguns valores de $q$ pequenos. Sabe-se que $c(5)=4$. O limite superior pode ser provado novamente usando esse método. Escolha $\mathcal{L}=$ $\{(0,2,3),(3,0,2),(2,3,0)\}$. Como $\mathcal{L}$ é invariante pela ação do 3 -ciclo $\varphi:\left(u_{1}, u_{2}, u_{3}\right) \mapsto\left(u_{2}, u_{3}, u_{1}\right)$, considere $N=\langle\varphi\rangle$ o subgrupo gerado por $\varphi$. A ação de $G=\langle\varphi\rangle \times K$ em $\mathcal{A}_{5}$ gera cinco órbitas. Visto que o estabilizador de um vetor $u$ é o subgrupo trivial, cada órbita $u^{G}$ tem doze elementos. Além disso, cada um dos representantes são cobertos por $\mathcal{L}$, como descrito abaixo

$$
\begin{array}{ll}
(0,1,2)=3(0,2,3)+3 e_{3} & (0,1,3)=3(0,2,3)+4 e_{3} \\
(0,1,4)=3(0,2,3) & (1,2,3)=1(0,2,3)+1 e_{1} \\
(1,3,2)=4(0,2,3)+1 e_{1}, &
\end{array}
$$

onde $e_{1}=(1,0,0), e_{2}=(0,1,0)$ e $e_{3}=(0,0,1)$. O limite $c(5) \leq 4$ segue do Teorema 7 .

\subsection{Aplicações numéricas}

Os limitantes superiores $c(8) \leq 6$ e $c(9) \leq 6$ são obtidos nessa seção, utilizando o Teorema 7 . Mas antes disso, uma forma de representar os elementos de um corpo finito $\mathbb{F}_{q}$ é discutida.

Denote os elementos não nulos de $\mathbb{F}_{q}$ como segue: Para todas as potências de primo $q$, o grupo multiplicativo $\mathbb{F}_{q}^{*}$ é cíclico e é isomorfo ao grupo aditivo $\mathbb{Z}_{q-1}$, pelo isomorfismo de $\mathbb{Z}_{q-1}$ em $\mathbb{F}_{q}^{*}$, dado por $\bar{x} \mapsto \xi^{x}$, onde $\xi$ denota um gerador arbitrário de $\mathbb{F}_{q}^{*}$. Então, a partir de agora considere $\mathbb{F}_{q}^{*}=\left\{\xi^{0}, \xi^{1}, \ldots, \xi^{q-2}\right\}$. A multiplicação em $\mathbb{F}_{q}^{*}$ segue a regra: $\xi^{x} \xi^{y}=\xi^{x+y}=\xi^{z}$, se e somente se, $z=x+y$ em $\mathbb{Z}_{q-1}$.

Exemplo 9. Tem-se que se 2 é um gerador do grupo multiplicativo de $\mathbb{Z}_{5}^{*}$, então podemos escrever $\mathbb{Z}_{5}=\left\{0,1,2^{1}, 2^{2}, 2^{3}\right\}$. Os vetores

$$
h_{1}=(1,1,1), h_{2}=\left(0,1,2^{2}\right), h_{3}=\left(1,2^{2}, 2^{2}\right) \text { e } h_{4}=(1,0,0),
$$

formam uma cobertura curta de $\mathbb{Z}_{5}^{3}$.

De fato, dado $u=\left(u_{1}, u_{2}, u_{3}\right)$ em $\mathbb{Z}_{5}^{3}$ um vetor arbitrário tem-se. Se $u$ tem alguma coordenada nula, então ele é coberto por $h_{2}=\left(0,1,2^{2}\right)$ e $h_{4}=(1,0,0)$. Por exemplo, se $u=\left(0, u_{2}, u_{3}\right)$, como $u_{2}\left(0,1,2^{2}\right)=\left(0, u_{2}, u_{2} 2^{2}\right)$ tem-se $h_{2}=\left(0,1,2^{2}\right)$ cobre $u$. Se $u$ tem pelo menos duas coordenadas iguais, então ele é coberto por $h_{1}=(1,1,1)$. Por exemplo se $u=\left(u_{1}, u_{1}, u_{3}\right)$, então como $u_{1}(1,1,1)=\left(u_{1}, u_{1}, u_{1}\right)$ segue-se $h_{1}=(1,1,1)$ cobre $u$. Agora, se $u$ tem as três coordenadas não nulas e distintas, então podemos supor sem perda de generalidade que $u$ é da forma $u=\left(1, u_{2}, u_{3}\right)$. Portanto, basta mostrar que os vetores $h_{1}, h_{2}, h_{3}$ e $h_{4}$ cobrem os vetores

$$
\left(1,2^{1}, 2^{2}\right),\left(1,2^{1}, 2^{3}\right),\left(1,2^{2}, 2^{1}\right),\left(1,2^{2}, 2^{3}\right),\left(1,2^{3}, 2^{1}\right),\left(1,2^{3}, 2^{2}\right) .
$$

Rapidamente pode-se observar que $h_{3}=\left(1,2^{2}, 2^{2}\right)$ cobre $\left(1,2^{1}, 2^{2}\right),\left(1,2^{2}, 2^{1}\right),\left(1,2^{2}, 2^{3}\right)$ e $\left(1,2^{3}, 2^{2}\right)$. Como $2^{1} h_{2}=\left(0,2^{1}, 2^{3}\right)$ e $2^{3} h_{2}=\left(0,2^{3}, 2^{1}\right)$ segue que $h_{2}=\left(0,1,2^{2}\right)$ cobre $\left(1,2^{1}, 2^{3}\right)$ e $\left(1,2^{3}, 2^{1}\right)$.

Proposição 10. O limite superior $c(8) \leq 6$ é válido.

Ideia da demonstração. Seja $\xi$ um gerador do grupo multiplicativo $\mathbb{F}_{8}^{*}$ e considere os vetores

$$
\begin{array}{lll}
h_{1}=(1,1,1), & h_{2}=\left(0,0, \xi^{1}\right), & h_{3}=\left(1, \xi^{1}, 0\right), \\
h_{4}=\left(1, \xi^{2}, \xi^{3}\right), & h_{5}=\left(1, \xi^{3}, \xi^{2}\right), & h_{6}=\left(\xi^{6}, \xi^{5}, 1\right) .
\end{array}
$$

É possível provar que $\mathcal{H}=\left\{h_{1}, \ldots, h_{6}\right\}$ é uma cobertura curta de $\mathbb{F}_{8}^{3}$, via Teorema 7 , conforme [6, Proposição 24].

Proposição 11. Temos $c(9) \leq 6$.

Ideia da demonstração. Considere $\xi$ um gerador do grupo multiplicativo $\mathbb{F}_{9}^{*}$ e os vetores

$$
\begin{array}{lll}
h_{1}=(1,1,1), & h_{2}=(1,0,0), & h_{3}=\left(0,1, \xi^{4}\right), \\
h_{4}=\left(1, \xi^{2}, \xi^{4}\right), & h_{5}=\left(1, \xi^{4}, \xi^{2}\right), & h_{6}\left(1, \xi^{6}, \xi^{6}\right),
\end{array}
$$

Assim $\mathcal{H}=\left\{h_{1}, \ldots, h_{6}\right\}$ é uma cobertura curta de $\mathbb{F}_{9}^{3}$. Ver detalhes em [6, Proposição 25]. 


\section{Conclusão}

Concluímos esse trabalho com a seguinte contribuição para o cálculo da função $c$.

Teorema 12. São válidos $c(7)=5, c(8)=6$ e $c(9)=6$.

Demonstração. É consequência imediata dos resultados anteriores. De fato, o limite inferior $c(7) \geq 5$ segue da Proposição 4, enquanto que o limite superior $c(7) \leq 5$ provém da desigualdade (2). O valor $c(8)=6$ é uma consequência imediata das Proposições 5 e 10. Obtém-se pelas Proposições 6 e 11 que $c(9)=6$.

\section{Referências}

[1] W.A. Carnielli, Hyper-rook domain inequalities, Stud. Appl. Math., 82 (1990), no. 1, 59-69.

[2] W.A. Carnielli, On covering and coloring problems for rook domains, Discrete Math., vol. 57 (1985), 9-16.

[3] G. Cohen, I. Honkala, S. Litsyn e A. Lobstein, Covering Codes, North-Holland, Amsterdam, (1997).

[4] M. Garey e D. Johnson, Computers and intractability: a guide to the theory of NPcompleteness, W.H.Freeman and Company, (1979).

[5] G. Kéri, Tables for bound on covering codes, homepage: http://www.sztaki.hu/ ${ }^{\sim}$ keri/ acessado (2014).

[6] A.N. Martinhão e E.L. Monte Carmelo, Intersecting families of extended balls in the Hamming spaces, http://arxiv.org/

[7] A.N. Martinhão e E.L. Monte Carmelo, Short covering codes arising from matchings in weighted graphs, Math. Comput., vol. 82 (2013), 605-616.

[8] C. Mendes, E.L. Monte Carmelo e M. Poggi, Bounds for Short Covering Codes and Reactive Tabu Search. Discrete Applied Mathematics, (2009), 522-533.

[9] E.L. Monte Carmelo, I.N. Nakaoka e J.R. Gerônimo, A covering problem on finite spaces and rook domains, Inter. J. Appl. Math., 20 (2007), 875-886.

[10] E.L. Monte Carmelo e I.N. Nakaoka, Short coverings in tridimensional spaces arising from sum-free sets, European J. Combin., 29 (2008), 227-233.

[11] O. Taussky e J. Todd, Covering theorems for groups, Ann. Soc. Polonaise Math. 21, (1948), 303-305. 